

SANDIA REPORT sAND82-1714 • Unlimited Release • UC-62

Printed September 1982

Dr. 911

\title{
Characterizing Solar Mirror Materials Using Portable Reflectometers
}

SAND- $-82-1714$

DE83 000804

\section{MASTER}

Richard B. Pettit

Prepared by

Sandia National Laboratories

Albuquerque, New Mexico 87185 and Livermore, California 94550

for the United States Department of Energy

under Contract DE-AC04-76DP00789

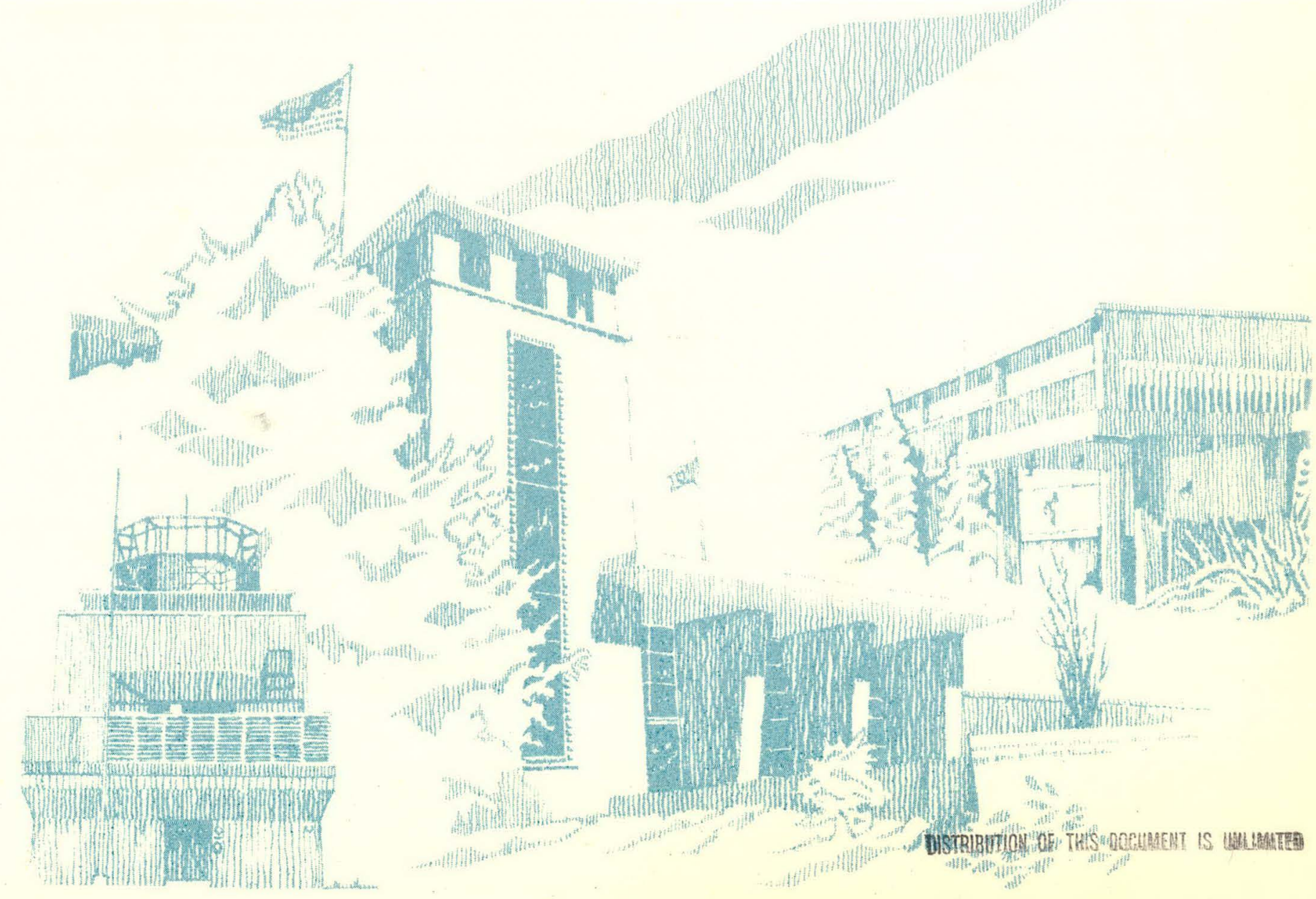




\section{DISCLAIMER}

This report was prepared as an account of work sponsored by an agency of the United States Government. Neither the United States Government nor any agency Thereof, nor any of their employees, makes any warranty, express or implied, or assumes any legal liability or responsibility for the accuracy, completeness, or usefulness of any information, apparatus, product, or process disclosed, or represents that its use would not infringe privately owned rights. Reference herein to any specific commercial product, process, or service by trade name, trademark, manufacturer, or otherwise does not necessarily constitute or imply its endorsement, recommendation, or favoring by the United States Government or any agency thereof. The views and opinions of authors expressed herein do not necessarily state or reflect those of the United States Government or any agency thereof. 


\section{DISCLAIMER}

Portions of this document may be illegible in electronic image products. Images are produced from the best available original document. 
Issued by Sandia National Laboratories, operated for the United States Department of Energy by Sandia Corporation.

NOTICE: This report was prepared as an account of work sponsored by an agency of the United States Government. Neither the United States Government nor any agency thereof, nor any of their employees, nor any of their ment nor any agency thereof, nor any of their employees, nor any of their
contractors, subcontractors, or their employees, makes any warranty, express or implied, or assumes any legal liability or responsibility for the accuracy, completeness, or usefulness of any information, apparatus, product, or process disclosed, or represents that its use would not infringe privately owned rights. Reference herein to any specific commercial product, process, or service by trade name, trademark, manufacturer, or otherwise, does not necessarily constitute or imply its endorsement, recommenda by the United States Govly its end, any age by the United States Government, any agency thereof or any of thei contractors or subcontractors. The views and opinions expressed herein do not necessarily state or reflect those of the United States Government, any agency thereof or any of their contractors or subcontractors.

Printed in the United States of America Available from

National Technical Information Service

U.S. Department of Commerce

5285 Port Royal Road

Springfield, VA 22161

NTIS price codes

Printed copy: A02

Microfiche copy: A01 
CHARACTERIZING SOLAR MIRROR MATERIALS USING PORTABLE REFLECTOMETERS

\begin{abstract}
Currently available portable instrumentation for hemispherical and specular reflectance measurements of solar mirror materials is discussed. Particular attention is given to the wavelength dependence of the measurement spectrum, which in most cases does not approximate a solar spectral distribution, and to other limitations of each instrument. Because a portable instrument is not available that can determine the solar averaged specular reflectance from a single measurement, two procedures are recommended for obtaining a reasonable estimate for this quantity using the existing portable equipment. Finally, future developments in this area are briefly discussed.
\end{abstract}

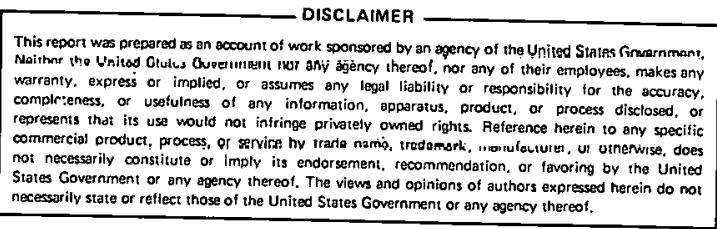

₹ 


\section{TABLE OF CONTENTS}

1.0 INTRODUCTION . . . . . . . . . . . . . . . . . 2

2.0 HEMISPHERICAL REFLECTANCE ................. 2

2.1 Devices and Services SSR Reflectometer ......... 3

2.2 Gier Dunkle MS-251 Solar Reflectometer . . . . . . . 4

3.0 SPECUlaR REFLECTANCE MEASUREMENTS . . . . . . . . . . . 6

3.1 Sandia Portable Specular Reflectometer . . . . . . 9

3.2 Devices and Services Portable Specular Reflectometer . . 13

4.0 RECOMMENDED MEASUREMENT PROCEDURE . . . . . . . . . . . . . 14

4.1 Procedure I.................. 14

4.2 Procedure II . . . . . . . . . . . 15

6.0 FUTURE DEVELOPMENTS . . . . . . . . . . . . . 16

7.0 CONCLUSIONS . . . . . . . . . . . . . . . . 17

8.0 APPENDIX . . . . . . . . . . . . . . . . . . . . 18

REFERENCES . . . . . . . . . . . . . . . . . . 20 


\subsection{Introduction}

Characterization of the reflectance properties of solar mirror materials requires the measurement of both the hemispherical and specular reflectance characteristics. The need to measure both of these quantities and their relationship to each other have been described in published reports.1 While these two reflectance properties can be adequately measured in the laboratory on moderately sized samples (up to approximately $25 \mathrm{~cm} \times 25 \mathrm{~cm}$ ), measurements $\because$ in the field on large samples using currently available portable instrumentation are limited. 'The purpose of this report is to discuss the measurement characteristics of these portable instruments and how these characteristics 1 imit the determination of a solar averaged specular reflectance value. Two procedures are recommended for estimating the solar averaged specular reflectance value based on measurements obtained using the available instruments. Since one objective of field measurements is to characterize the specular reflectance changes that occur with outdoor exposure, a distinction is drawn between measuring changes in the intrinsic reflectance properties of the mirror and changes caused by accumulated dust particles. The usefulness of present instrumentation in these areas is outlined.

\subsection{Ilemispherical Reflectance}

Hemispherical reflectance is defined as the ratio of the radiation reflected from a surface into a hemisphere to the radiation incident on that surface. ${ }^{2}$ Although the hemispherical reflectance contains no information concerning the angular distribution of the reflected radiation, it does represent the upper Iimit for the specular reflectance for a material.

While the hemispherical reflectance is a function of the incident angle, for typical solar materials, this dependence is small for angles up to 60 
degrees from normal.3 Therefore, measurements limited to incident angles less than 30 degrees from normal should all provide equivalent values.

All portable instruments designed to measure the solar averaged hemispherical reflectance, $R_{S}(2 \pi)$, contain their own light source. Typically, these sources do not provide a good match to a solar spectral distribution. Therefore, it is important to understand the spectral characteristics of each instrument and how the measurement spectrum differs from a typical solar spectral distribution. These differences can lead to appreciable errors in the measured solar averaged hemispherical reflectance value, depending upon the reflectance characteristic of the mirror being measured. The measurement characteristics of two currently available portable instruments designed to measure $R_{S}(2 \pi)$ are discussed below. 2.1 Devices and Services SSR Reflectometer: The Devices and Services (D\&S) Solar Spectrum Reflectometer (SSR) hemispherically illuminates a sample placed over the measurement port and measures the amount of radiation reflected from the sample at a 20 degree angle using four separate detector/filter combinations. The four detector/filters cover distinct wavelength regions across the solar spectrum as shown in Fig. 1a. The output of each detector is weighted according to the portion of the solar spectral distribution to be matched. By summing these weighted outputs, an approximation to the solar averaged hemispherical reflectance is obtained.

While the overall wavelength response of the instrument provides a good match to a solar spectral distribution (see Fig. 1b), other problems have been found when measuring second surface solar mirror materials; modification of the measurement head of this instrument has improved the measurement accuracy for such mirrors.4 without this modification, the instrument significantly underestimates the actual solar averaged hemispherical reflectance for second surface mirrors. The problem arises from the fact that, for second surface 




WAVELENGTH, nII

Figure 1. (a) Wavelength response of the four detector/filter combinations used in the Devices and Services SSR reflectometer.

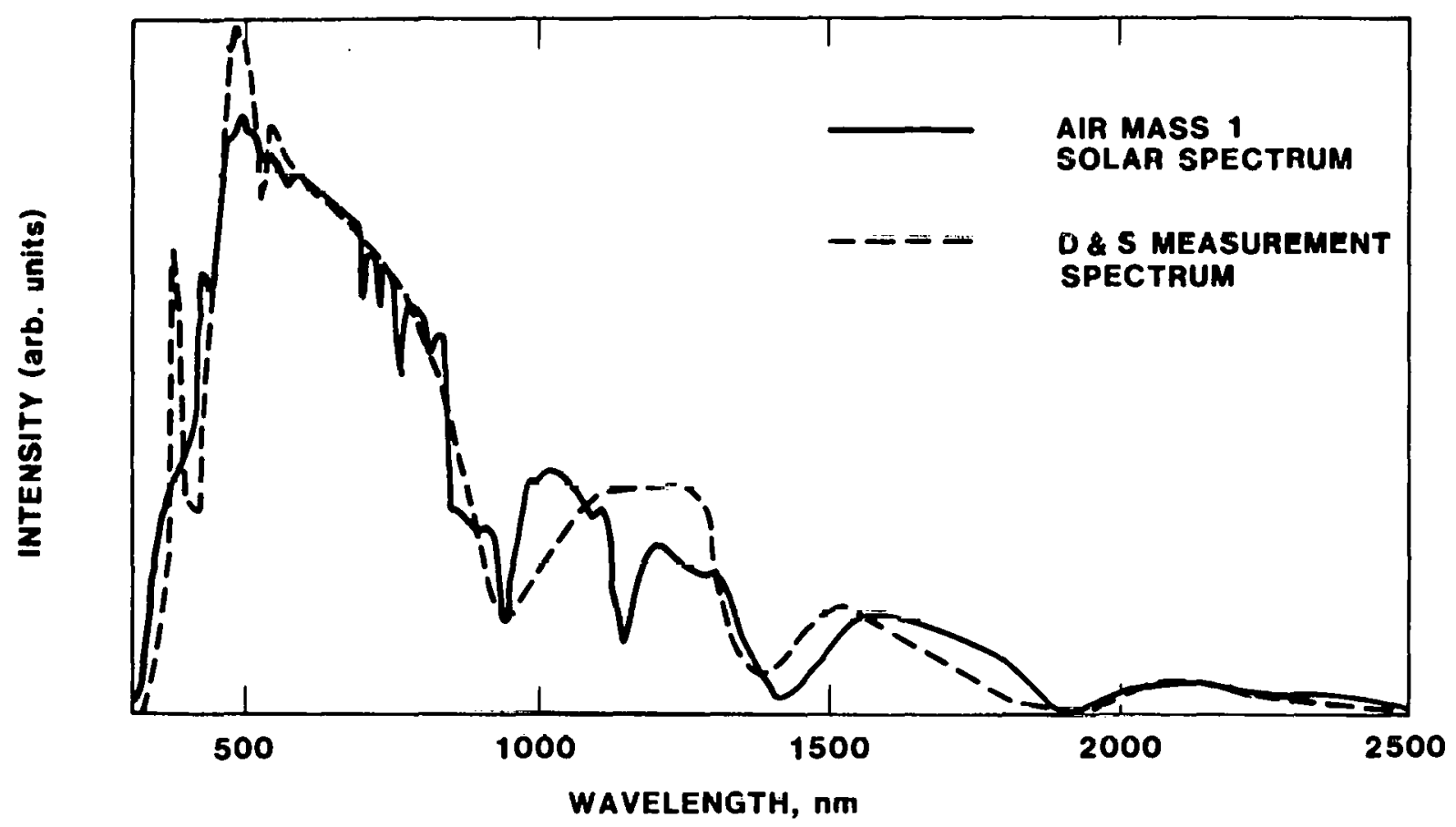

(b) The effective measurement spectrum of the SSR reflectometer as compared to an air mass one solar spectral distribution. 
mirrors, the reflecting surface (metal layer) is displaced away from the plane of the measurement port in the instrument. The greater the thickness of the transparent protective layer (typically glass or plastic), the greater this displacement and the larger the measurement error. After modifying the instrument as described in Ref. 4, the measured solar reflectance is only 58 below the true value for a second surface glass mirror $6 \mathrm{~mm}$ thick. This value is compared to a reflectance decrease of over 208 before the modifications. This smaller reduction in the measured reflectance value can be corrected using an easily generated correction curve (see Ref. 4). Using this curve, the solar averaged hemispherical reflectance of mirrors varying in thickness from 0 to 6 $\mathrm{mm}$ can be determined (after correction) to an accuracy of \pm 0.008 reflectance units $(1008$ reflectance $=1.000$ reflectance units $)$

Finally, it should be pointed out that the reflectance properties of a sample can also be measured with each individual detector/filter in order to obtain an indication of the wavelength dependence of the reflectance properties. This measurement technique will be discussed later as one of the recomnended procedures for determining the solar averaged specular reflectance properties. 2.2 Gier Dunkle MS-251 Solar Reflectometer: Gier Dunkle Instruments, Santa Monica, California (now part of Dynatech) manufactures a solar spectrum reflectometer, Model Ms-2bl. The operating characteristics of this instrument have previously been described. 5 In this instrument, the output of a xenon lamp is directed onto a sample and the amount of reflected energy measured using an integrating sphere. By comparing the intensity of radiation reflected from the sample to the intensity of the incident radiation, the hemispherical reflectance of the sample is obtained. The measurement spectrum, shown in Fig. 2 , is approximately equal to the spectral output of the xenon lamp that contains many large intensity peaks from 800 to 1100 nanometers. The differences between 


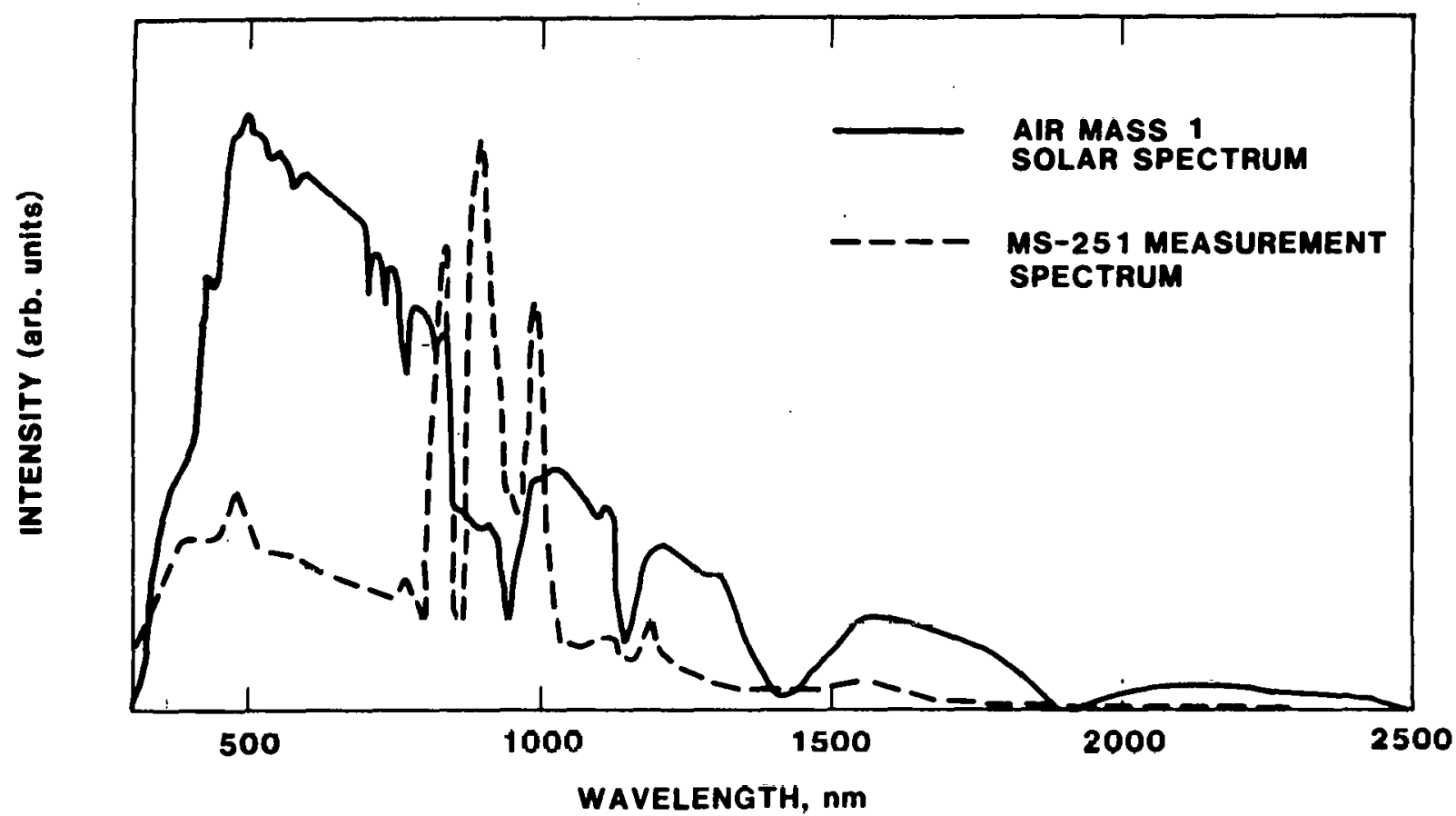

Figure 2. Measurement spectrum of a Giex-Dunkle Solar Reflectometex, Model MS251. Also shown is an air mass one solar spectral distribution.

this measurement spectrum and the solar spectral distribution can lead to errors in the measured solar averaged hemispherical reflectance value. The magnitude of these errors has not been quantified for typical solar mirror materials.

In addition, the MS-251 also underestimates the reflectance values measured for second surface mirrors. This occurs because some of the radiation reflected at the metal layer of a second surface mirror does not enter the measurement cavity (sphere) but is clipped by the measurement port. Correction of this problem would parallel the modifications performed on the D\&S SSR instrument, but no correction has been attempted for this instrument. Correction curves as a function of mirror thickness could also be obtained for the MS-251 in a manner similar to the techniques used for the D\&S SSR instrument (see Ref. 4).

\subsection{Specular Reflectance Measurements}

It has been found that the specularly reflected beam profile of solar mirror materials can be adequately described by a normal distribution of the form 


$$
R(\Delta \theta)=\frac{R_{0}}{2 \pi \sigma_{0}^{2}} \exp \left[\frac{-\Delta \theta^{2}}{2 \sigma_{0}^{2}}\right]
$$

where $\sigma_{0}$ is the dispersion of the distribution, $\Delta \theta$ is the angle measured from the specular direction and $R_{0}$ is the integrated reflectance value at the wavelength of interest. ${ }^{1}$ Typically, $\mathrm{R}_{\mathrm{O}}$ is equal to the hemispherical reflectance. A determination of the dispersion $\left(\sigma_{0}\right)$ requires reflectance measurements using a bidirectional reflectometer over a range of angular apertures, typically from $1 \mathrm{mrad}$ to 15 or $30 \mathrm{mrad}$, and computer analysis of the resulting data. 6 Presently available portable instruments are not capable of this type of detailed data acquisition.

In a solar concentrator, the reflectance quantity of interest is the amount of reflected sunlight that is incident on the receiver surface. A detailed discussion of how the specular reflectance properties are utilized in calculating the solar collector performance is presented in Ref. 7. Briefly, the quantity of interest is the integral of equation (1) out to an angular size, $\theta$, which represents the angular size of the receiver surface. This quantity, which is termed the cumulative reflectance $R^{\prime}(\theta)$, is given by 8

$$
\begin{aligned}
R^{\prime}(\theta) & =\int_{0}^{\theta} 2 \pi \Delta \theta R(\Delta \theta) d \Delta \theta \\
& =R_{0}\left[1-\exp \left(\frac{-\theta^{2}}{2 \sigma_{0}^{2}}\right)\right]
\end{aligned}
$$

Note that when $\theta \gg \sigma_{0}, R^{\prime}(\theta)$ is equal to $R_{0}$, as expected.

While the parameters in equations (1) and (2) are valid at specific wavelengths, the equations themselves can be used to describe the solar averaged specular reflectance properties. In this case, $R_{O}$ is replaced by its solar averaged value, $R_{S}$, and $\sigma_{O}$ is replaced by a solar averaged dispersion, $\sigma_{S}$. 
As discussed below, currently available portable specular reflectometers cannot measure $R_{S}^{\prime}(\theta)$.

In the special case of silvered glass mirrors, the dispersion $\sigma_{0}$ has been found to be always less than or equal to $0.5 \mathrm{mrad}$, which is the resolution of the laboratory measurement. Thus for collection angles $\theta \geq 2 \mathrm{mrad}$, the cumulative reflectance is equal to the hemispherical reflectance and is independent of the size of the collection aperture $\theta$ used in the measurement.

Measurements have shown ${ }^{9}$ that accumulated dust on the surface of a mirror does not significantly change the dispersion $\sigma_{0}$, but only reduces the intensity $R_{0}$ of the specular beam (see Eq. (1)). This means that the fractional decrease in the cumulative reflectance due to dust accumulation is the same at all angular apertures, $\theta$. Thus, if a measurement is designed to determine the decrease in the cumulative reflectance due to dust accumulation, the size of the measurement angular aperture used in the portable specular reflectometer is not important. However, other changes in $\mathrm{R}^{\prime}(\theta)$ due to outdoor exposure may be a function of the measurement angular aperture (i.e., $\sigma_{0}$ may change).

The reduction in the specular reflectance due to soiling is a function of wavelength. Measurements have shown that the specular reflectance loss (which is defined as the difference between $R_{0}$ measured before and after soiling) increases with decreasing wavelength over the solar spectral reginn. In addition, the wavelength dependence changes considerably with the exposure conditions. 9 Typical variations in the wavelength dependent behavior for silvered glass mirrors after various lengths of exposure in Albuquerque, NM, are shown in Fig. 3 . For the data shown, the wavelength dependence of the specular reflectance loss caused by the spectral reflectance characteristics of the silvered glass mirrors has been removed, as discussed in Ref. 9 , in order to emphasize the wavelength dependence resulting only from the soiling. In addition, all curves 




Figure 3. Wavelength dependence of the specular reflectance loss caused by dast accumulation auriny outdoor exposure. All curves have been normalized to pass through 1.00 at $500 \mathrm{~nm}$ (see Ref. 9). The time of outdoor exposure is listed next to each curve.

have been normalized to pass through the value 1.00 at $500 \mathrm{~nm}$ in order to highlight their different wavelength behavior. Clearly, a reflectance measurement over the complete solar spectrum is required in order to obtain an accurate indication of the solar averaged specular reflectance loss because of these wavelength and exposure time variations. Errors in the specular reflectance loss as a result of this wavelength dependence are discussed below for the available portable specular reflectometers.

\subsection{Sandia Portable Specular Reflectometer: In early 1978, J. M. Freese} developed a portable specular reflectometer whose optical design was based on 
the laboratory bidirectional reflectometer discussed earlier.10 7 A schematic of the optical arrangement is shown in Fig. 4. Cumulative reflectance values obtained with the portable instrument agreed with data obtained using the laboratory bidirectional reflectometer to within \pm 0.005 reflectance units. 10

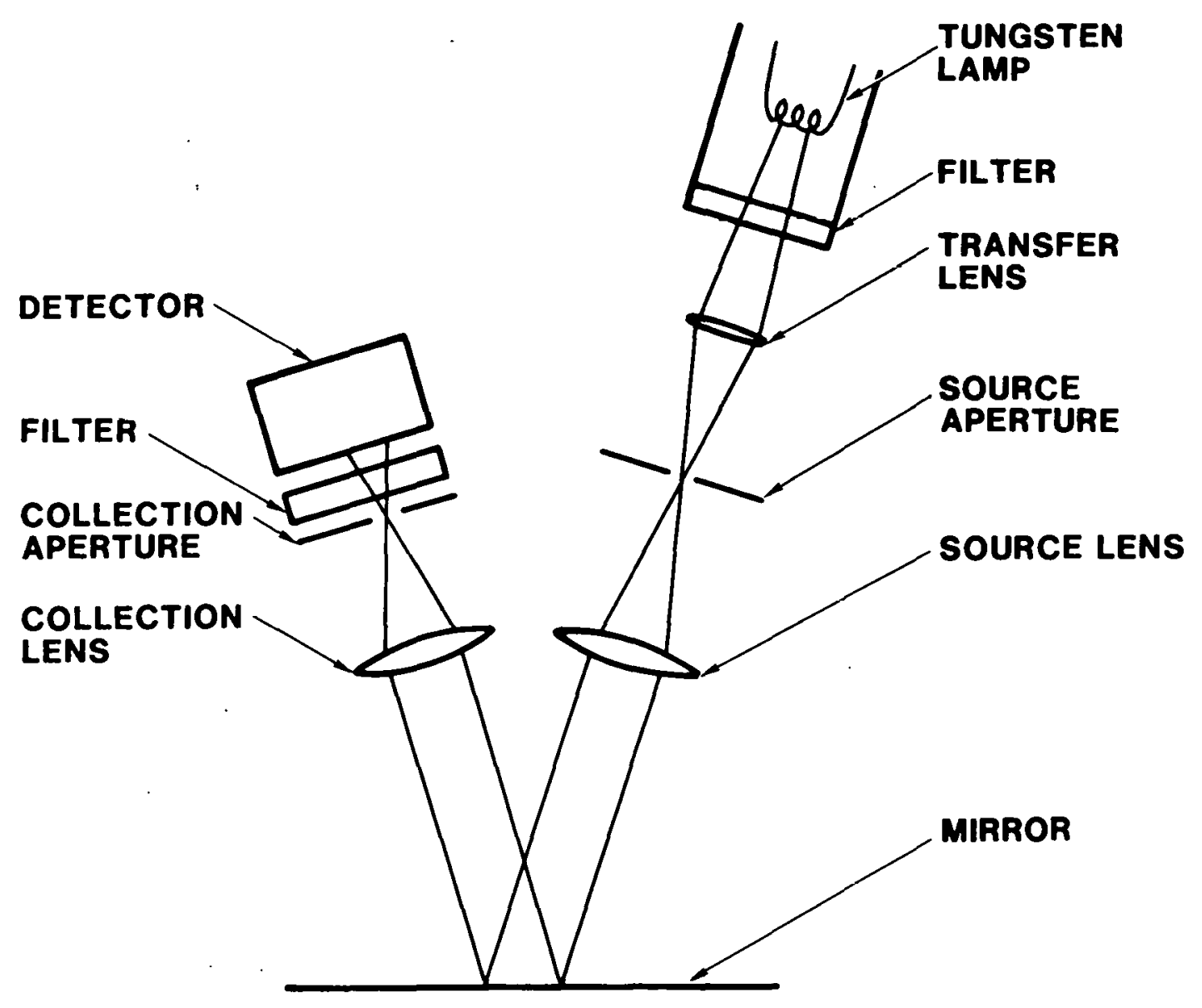

Figure 4. Schematic optical diagram of a portable specular reflectometer showing the location of the collimation and collection lenses, filters, the light source and the detector.

Because of the success of this first prototype, a second generation instrument ${ }^{11}$ was built as shown in Fig. 5. This instrument incorporated several improvements over the first prototype including: (1) A larger diameter measurement beam (10 mm vs $5 \mathrm{~mm}$ ); (2) Reduced weight from 6.5 lbs to $3.51 \mathrm{bs}$;

(3) The addition of adjustable apertures for ease of alignment; (4) Ability to 
measure specular transmittance properties as well as specular reflectance properties; (5) Improved $\mathrm{X}-\mathrm{Y}$ beam tilting adjustments that are used for alignment. A complete set of drawings for this instrument was also prepared.

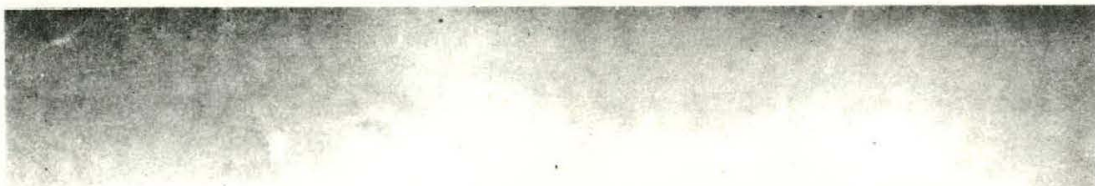

D \& S REFLECTOMETER

SANDIA REFLECTOMETER AND POWER SUPPLY

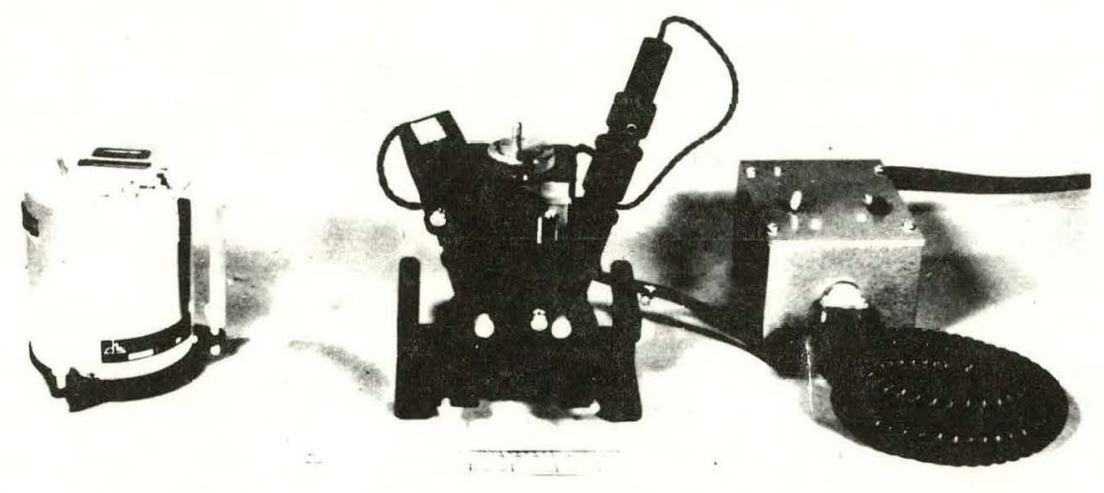

Figure 5. Photograph showing the Sandia-designed second generation portable specular reflectometer and power supply (right) and the new Devices and services portable specular reflectometer (left).

The measurement spectrum of this instrument was defined primarily by the spectral characteristics of several internally mounted filters, as well as the optical characteristics of the light source (tungsten lamp) and detector (silicon photocell). The wavelength dependence of the measurement spectrum was measured and is shown in Fig. 6. Note that the spectrum extends from $450 \mathrm{~nm}$ to $750 \mathrm{~nm}$ and peaks near the maximum of the solar spectral distribution (compare with Fig. 1b). Since this measurement spectrum does not correspond to a solar spectral distribution, the instrument cannot determine the solar averaged cumulative reflectance properties. The limited measurement spectrum also affects the accuracy of changes measured in the specular reflectance due to dust accumulation. From 
the data shown in Fig. 3, the sample represented by the 10-month curve would cause the largest error in measured cumulative reflectance loss using the Sandia reflectometer as compared to the actual solar averaged cumulative reflectance 10ss. For this curve, the Sandia instrument will measure a cumulative reflectance loss that is $20 \%$ higher than the actual solar averaged loss. For example, if the solar averaged cumulative reflectance loss were 0.100 reflectance units, the Sandia instrument would measure a loss of 0.120 . Note that the potentially large percent error $(20 \%)$ in the Sandia instrument does not translate into substantial reflectance errors for small to moderate cumulative reflectance losses. For this reason, this limitation is considered only a minor problem.

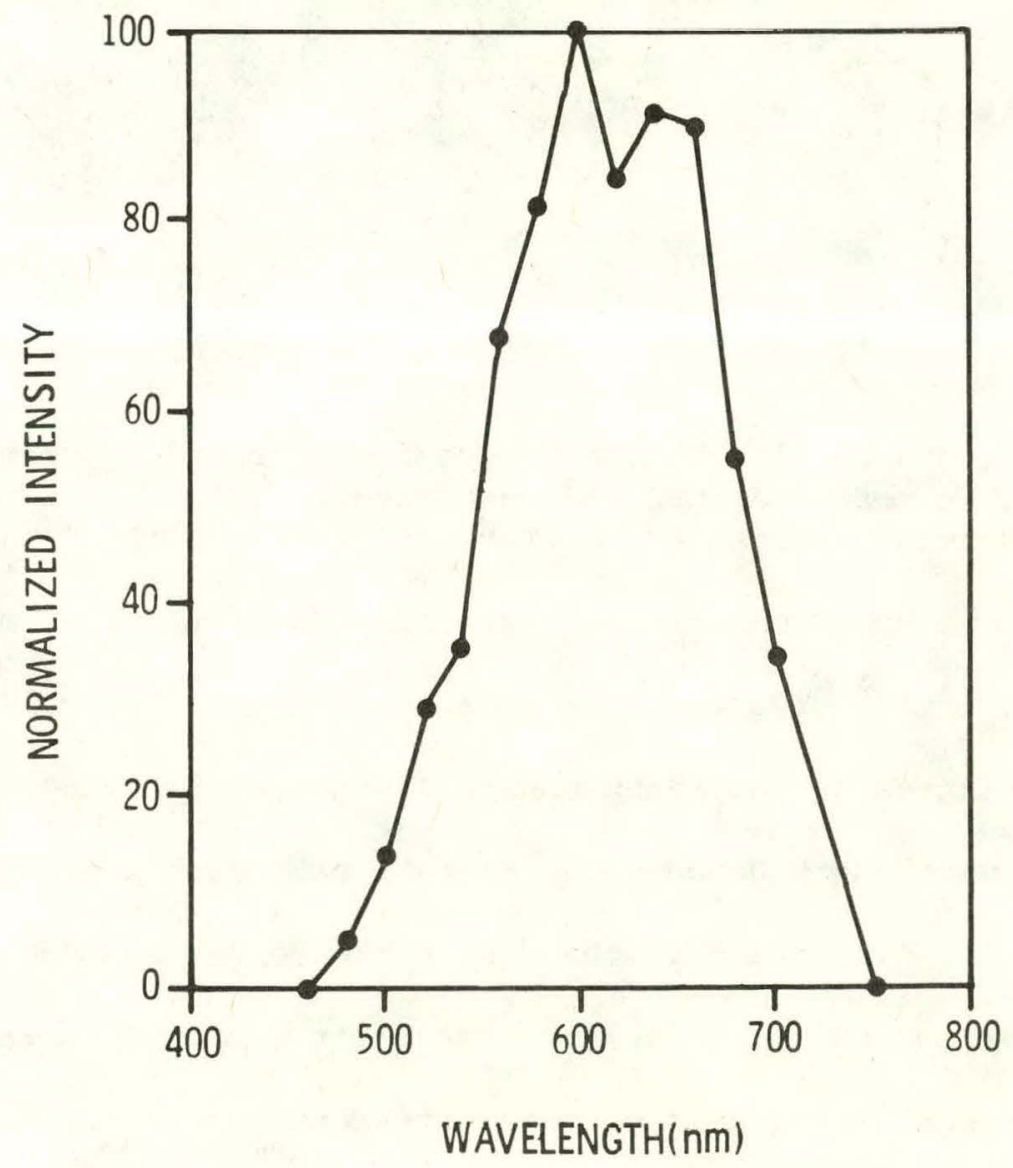

Figure 6. The wavelength dependence of the measurement spectrum of the Sandia second generation portable specular reflectometer (see Ref. 11). 
3.2 Devices and Services Portable Specular Reflectometer: D\&S, under contract to sandia, has developed a portable specular reflectometer based on the previously discussed sandia instrument. A picture of the instrument is shown on the left in Fig. 5. While this new instrument uses the same optical design as the Sandia reflectometer, it incorporates several improvements: (1) The electronics are entirely contained within the measurement head and are battery operated; thus the instrument is completely portable. (2) Three different collection apertures can be easily selected ranging in size from $\theta=7.5 \mathrm{mrad}$ to $\theta=23 \mathrm{mran}$. (3) The light source is chopped so that the instrument is not sensitive to direct background light. Thus, the instrument can be used outdoors in direct sunilight without affecting the reflectance readings. (4) A viewing eyepiece is installed in the instrument so that an image of the reflected beam can be viewed directly.

This reflectometex uses a light emitting diode (LED) as the light source. The measurement spectrum is thus limited to a narrow $50 \mathrm{~nm}$ wide band centered at $660 \mathrm{~nm}$. While this obviously precludes measurement of the solar averaged cumulative reflectance, it does reduce the errors associated with the wavelength dependent scattering caused by dust accumulation. For the 10 month sample data shown in Fig. 3, the D\&S reflectometer will measure a cumulative reflectance loss only 58 higher than the true solar averaged loss. Thus a solar average cumulative reflectance loss of 0.100 reflectance units will be measured as 0.105 with this instrument.

After a 15-minute warmup period, this reflectometer performs as well as the Sandia instrument. However, because the measurement optics are completely enclosed, the instrument is not capable of the direct specular transmittance measurements that are possible with the Sandia portable reflectometer. 


\subsection{Recommended Measurement Procedure}

The currently available portable specular reflectometers cannot directly provide a value for the solar averaged cumulative reflectance, $\mathbf{R}_{\mathbf{s}}^{\prime}(\theta)$. Therefore, two procedures are recommended for obtaining a reasonable estimate for $R_{S}^{\prime}(\theta)$ using the existing portable instrumentation described above.

It should be reemphasized that, for clean silvered glass mirrors, the solar averaged hemispherical reflectance, $R_{\mathbf{s}}(2 \pi)$, is always equal to the solar averaged cumulative reflectance, $R_{s}^{\prime}(\theta)$, for $\theta \geq 2 \operatorname{mrad}$. Therefore, $a$ portable hemispherical solar reflectometer can be used to determine the solar averaged cumulative reflectance properties of a silvered glass mirror before any outdoor exposure. After outdoor exposure, a portable specular reflectometer can be used to determine the effect of dust accumulation following one of the procedures outlined below.

\subsection{Procedure I}

The easiest and most straightforward procedure for determining the solar averaged cumulative reflectance involves first measuring the solar averaged cumulative reflectance using laboratory instrumentation on a small sample of the mirror material of interest. Details for obtaining a vâlue of $R_{S}^{\prime}(\theta)$ in the laboratory are outlined in the Appendix. Next, the rumulativn roflcotance value, $R$ (port), is measured for the same small sample using a portable specular reflectometer. These two values are used to generate a correction factor, $c$, given by

$$
C=\frac{R_{S}^{\prime}(\theta)}{R(\text { port })}
$$

Multiplication of $R$ (poxt) by $C$ results in the value $R_{S}^{\prime}(\theta)$. Thus, subsequent measurements of the same material using the portable specular reflectometer can be converted to a solar averaged values by mulliplication using the constant C. 
For this calculational procedure to remain valid as the sample ages, the assumption is made that any changes in the cumulative reflectance properties over the wavelength band of the portable reflectometer, occur equally across the entire solar spectrum. For dust accumulation, this is not usually the case, as shown in Fig. 3. However, for the D\&S specular reflectometer, the above procedure leads to errors less than +0.01 reflectance units for losses up to 0.20. For the Sandia portable specular reflectometer, the maximum error is +0.04 reflectance units for losses up to 0.20 . For other changes in the cumulative reflectance properties, the resulting measurement errors may be unknown.

\subsection{Procedure II}

The alternate procedure utilizes hemispherical reflectance measurements obtained using the D\&S portable hemispherical reflectometer (SSR). This procedure can be used when a laboratory measurement of $R_{s}^{\prime}(\theta)$ for the same material is not available. In this procedure, a value of $R_{s}(2 \pi)$ is first obtained using the D\&S SSR. Next the hemispherical reflectance is measured using only the RED detector/filter combination of the SSR (see Fig. 1) to obtain a value $R_{\text {RED }}(2 \pi)$. Note that the wavelength response of this detector/filter peaks at $660 \mathrm{~nm}$, which is the same as the peak wavelength of the D\&S portable specular reflectometer. By comparing $\operatorname{R}_{\mathrm{RED}}(2 \pi)$ with the reflectance value measured with the D\&S portable specular reflectometer, $R_{\text {port }}(\theta)$, the relative amount of scattered radiation, $\Delta R$, in the wavelength region near $660 \mathrm{~nm}$ cain be calculated as

$$
\Delta R=\frac{R_{R F \cap}(2 \pi)-R_{\text {mrrt }}(\theta)}{R_{R E D}(2 \pi)} .
$$


By reducing $R_{S}(2 \pi)$ by the same fractional amount $\Delta R$, a reasonable estimate of the solar average cumulative reflectance can be obtained. Thus,

$$
\begin{aligned}
R_{S}^{\prime}(\theta) & =R_{S}(2 \pi)-\Delta R \cdot R_{S}(2 \pi) \\
& =R_{S}(2 \pi)(1-\Delta R) \\
& =R_{S}(2 \pi) \cdot \frac{R_{\text {port }}(\theta)}{R_{\text {RED }}(2 \pi)} .
\end{aligned}
$$

Again, this value is subject to similar assumptions as in the first procedure.

\subsection{Future Developments}

Clearly, a portable specular reflectometer is needed which can provide a solar aver aged cumulative reflectance value from a single measurement. The development of such an instrument must overcome several potential problems. First, the instrument measurement spectrum must match a solar spectral distribution. While xenon lamps filtered to remove the large intensity peaks near $1000 \mathrm{~nm}$ may approximate this distribution, they are usually large, require substantial electrical power, and their output is not stable with time. Secondly, the focal characteristics of currently used glass lenses change with wavelength. This leads to variations in the effective size of the measurement angular aperture with wavelength that may not be acceptable. Therefore, more expensive reflecting optics may have to be utilized in the future. Finally, there are very few detectors available that are sensitive across the entire solar spectrum (roughly 300 to $2000 \mathrm{~nm}$ ). Acceptable detectors would include thermopiles and pyroelectric devices. The use of several detector/filter combinations, as in the D\&S SSR instrument, is complicater by the fact that each detector must view the same measurement angular aperture. Several detector/filter combinations may also substantially increase the size, weight and power requirements of the instrument. Therefore, before an improved reflectometer is considered, the 
needs of the solar program and the limitations imposed by the presently available portable instrumentation and approximate measurement procedures should be carefully weighed.

\subsection{Conclusions}

Portable reflectometers are available for obtaining either hemispherical or specular reflectance information. In the case of hemispherical reflectometers, the instrumentation can provide accurate values for the solar averaged hemispherical reflectance, $R_{S}(2 \pi)$, if properly corrected. However, portable instrumentation is currently not available that can measure the solar averaged cumulative reflectance directly. A realistic estimation of this quantity can he obtained using one of two proposed measurement procedures. One procedure relies on previous measurements of the solar averaged cumulative reflectance using laboratory equipment. Alternatively, a measurement of both the hemispherical and specular reflectance values over the same wavelength region using the available portable instrumentation can be combined to estimate the solar averaged cumulative reflectance as described in the text.

For monitoring the effect of dust accumulation on the cumulative reflectance of solar mirror materials, the Devices and services portable specular retlectometer introduces less error in determining the solar averaged cumulative reflectance loss than the Sandia designed portable instrument. This results from the different wavelength measurement regions of the two instruments. Clearly, an understanding of the operational characteristics and limitatiuns of Llie available portable specular reflectometers is essential to using and interpreting their measured reflectance values correctly. 


\subsection{Appendix}

The purpose of this appendix is to discuss the determination of the solar averaged cumulative reflectance using laboratory instrumentation. The solar averaged cumulative reflectance properties can be adequately described by the equation

$$
R_{S}^{\prime}(\theta)=R_{S}\left[1-\exp \left(\frac{-\theta^{2}}{2 \sigma_{S}^{2}}\right)\right]
$$

This is identical to equation (2) except that $R_{0}$ and $\sigma_{0}$ have been replaced by their solar averaged values.

Currently, laboratory instruments that can measure the specular reflectance characteristics of solar mirrors are limited to measurements at a single wavelength (usually because they use a laser sourcell,12) or are limited to providing measurements at only discrete wavelengths. Therefore these data must be "averaged" in order to determine suitable values for $R_{S}$ and $\sigma_{S}$ in equation (la). Instead of obtaining cumulative reflectance data at a variety of wavelengths, the following procedure has been used.

First the cumulative reflectance $R^{\prime}(\theta)$ is measured at one wavelength, typically near $500 \mathrm{~nm}$ (the peak of the solar spectrum) as a function of $\theta$. The resulting curve is fit with the expression in equation (2) to determine $R_{0}$ and $\sigma_{O^{*}}$ The value of $R_{O}$ is $110 \mathrm{~L}$ set equal to the nemispherical reflectance value, $R_{\lambda}(2 \pi)$, measured at the same wavelength. The value determined for $R_{0}$ should be less than or equal to $R_{\lambda}(2 \pi)$. Note that the cumulative reflectance results are fit with a single normal distribution. This is done here even though earlier work has shown that, for some materials, a better fit can be obtained using the sum of two normal distributions. 6 The use of two distributions results in determining two sets of values $\left(R_{1}, \sigma_{1}\right)$ and $\left(R_{2}, \sigma_{2}\right)$. If the cumulative reflectance data do not closely match. a single 
distribution, then an alternative procedure must be used. However, if the reflectance data approximate a single distribution, the assumption is then made that $\sigma_{0}$ does not change appreciably with wavelength so that $\sigma_{S} \approx \sigma_{0}$. This assumption can be easily checked by measuring $R^{\prime}(\theta)$ at another wavelength, calculating $R_{0}^{\prime}$ and $\sigma_{0}^{\prime}$, and comparing $\sigma_{0}$ to $\sigma_{0}^{\prime}$. Significant differences between the $\sigma$ values mean that alternative averaging procedures need to be considered on an individual material-by-material basis.

If $\sigma_{0} \approx \sigma_{0}^{\prime}$ (which is usually the case $^{6}$ ), then the value $\mathrm{R}_{\mathrm{o}}$ should be compared to the hemispherical reflectance value, $R_{\lambda}(2 \pi)$, at the same wavelength. If $R_{0} \approx R_{\lambda}(2 \pi)$, the specular and hemispherical reflectance values are equivalent, and $R_{S}^{\prime}(\theta)$ is given by settlny $R_{s}=R_{s}(2 \pi)$ and $\sigma_{S}=\sigma_{0}$ in equation (1.a). If $R_{0}<R_{\lambda}(2 \pi)$, the amount of scattering $\Delta R_{\lambda}=R_{\lambda}(2 \pi)-R_{0}$ is calculated. The wavelength dependence of $\Delta \overline{\mathrm{R}}_{\lambda}$ has been found to be the sallte as lhe wave-length dependence of the diffuse reflectance. 1 (The diffuse reflectance is defined as the amount of reflected radiation that is outside a specified cone angle that is centered in the specular direction. The diffuse reflectance is measured using an integrating sphere as discussed in Ref. 9.) Thus a solar averaged value for $\Delta R$ can be calculated using the solar averaged diffuse reflectance, $R_{S}$ (diffuse), and the diffuse reflectance value, $R_{\lambda}$ (diffuse), measured at the same wavelength as $\Delta \mathrm{R}_{\lambda}$ using the following equation:

$$
\Delta R_{S}=\Delta R_{\lambda} \cdot \frac{R_{s}(\text { diffuse })}{R_{\lambda} \text { (diffuse) }}
$$

Finally, $\mathrm{R}_{\mathbf{S}}^{\prime}(\theta)$ is determined by setting

$$
\begin{aligned}
& R_{S}=R_{S}(2 \pi)-\Delta R_{S} \\
& \sigma_{S}=\sigma_{0}
\end{aligned}
$$

in equation (1a). 
1. R. B. Pettit and E. P. Roth, "Solar Mirror Materials: Their Properties and Uses in Solar Concentrating Collectors," in Solar Materials Science, Ed. by L. E. Murr, Academic Press, New York (1980).

2. See "Thermal Radiative Properties - Metallic Elements and Alloys," Vol. 8, Thermophysical Properties of Matter (Ed. by Y. S. Touloukian and D. P. Dewitt), pp. 1a-47a, Plenum, New York (1970).

3. D. N. Glidden and R. B. Pettit, "Specular Reflectance Properties of Solar Mirrors as a Function of Incident Angle" in Proceedings 1974 IsEs Congress, May 28-June 1, 1979, Atlanta, Georgia (pp. 1304-1308).

4. A. R. Mahoney, "Solar Hemispherical Reflectometer Modification for Second Surface Mirror Measurements," Sandia National Laboratories Report, SAND 820934 (May, 1982).*

5. R. B. Pettit, "Evaluation of Portable Optical Property Measurement Equipment for Solar Selective Surfaces," Engineering for Power 100, 489 (1978).

6. R. B. Pettit, "Characterization of the Reflected Beam Profile of solar iMirror Materials," Solar Energy 19, 733 (1977).

7. R. B. Pettit, C. N. Vittitoe and F. Biggs, "Simplified Calculational Procedure for Determining the Amount of Intercepted Sunlight in an Imaging Solar Concentrator," Solar Energy Engineering (submitted).

8. In this equation, $\Delta \theta$ is assumed small (<100 mrad) so that $\Delta \theta \cong \sin \Delta \theta$. The correct equations are contained in Ref. 7 .

9. R. B. Pettit and J. M. Freese, "Wavelength-Dependent Scattering Caused by Dust Accumulation on Solar Mirrors," Solar Energy Matexials 3 , 1 (1980).

10. J. M. Freese, "Development of a Portable Specular Reflectometer for Field Measurements of Solar Mirror Materials," Sandia National Laboratories Report, SAND 78-1918 (October 1978).*

11. T. M. Freese, "Ihe Development of a Second Generation Portable Specular Reflectometer," in Proceedings of Line-Focus Solar Thermal Energy Technology Development Conference, Sept. 9-11, 1980, Albuquerque, NM, pp. 467-476.

12. M. A. Lind, J. S. Hartman and H. L. Hampton, "Specularity Measurements for Solar Materials," in Proceedings SPIE, Vol. 161, Optics Applied to Solar Energy IV, pp. 98-105 (1978).

13. R. C. Zentner, "Performance of Low-Cost Solar Reflectors for Transmitting Sunlight to a Distant Collector," Solar Energy 19, 15 (1977).

*Available from: National Technical Information Services

U. S. Department of Commerce

5285 Port Royal Road

Springfield, VA 22161 
Distribution: (UC 62)

AAI Corporation

P. O. Box 6767

Baltimore, Maryland 21204

Attn: G. Ronald Christ

Harold Wilkening

Aluminum Company of America

Alcoa Technical Center

7 th Street Road

Route 780

Alcoa Center, PA 15069

Attn: John Powers

Burton A. Benson

Decorative Products Div.

3M Company

3M Center - Bldg. 209-2N

St. Paul, Minnesota 55101

Devices and Services

10,911 Dennis Road, Suite 405

Dallas, TX 75229

Attn: Charles Moore (2)

Harold Blum

Harry Dursch

Boeing Engineering and

Construction

P. O. Box 3707

Seattle, Washington 98124

Ford Aerospace and Communication Corporation

Western Development Laboratory Division

3939 Fabian way

Paln Altn, California 91304

Attn: John Nitchey

Richard A. Hays

White Sands Solar Furnace

WSMR, STEWS-TE-AN

White Sands, New Mexico 88002

Mr. E. W. Hoffman

Solar Systems Tndustries, Ltd.

No. 2-11771 Horseshoe Way

Richmond B.C. V7A 4S5

CANADA
Jacques L. Hull

Acurex Corporation

$485 \mathrm{Clyde}$ Avenue, MS 8-8800

Mountain View, California 94042

Joe A. Hutchison

Solar Kinetics, Inc.

3300 Century Circle

Dallas, Texas 75060

Kevin MacDonald

General Electric Company

Advanced Energy Program Department

720 Vandenberg Road, Bldg. B

King of Prussia, PA 19406

Keith Masterson

SERI

1617 Cole Blvd.

Golden, Colorado 80401

Stan Moore

LASL, Group Q-11

P. O. Box 1663, MS-571

Los Alamos, New Mexico 87545

Virginia L. Morris

McDonnell-Douglas

MS 22-2

5301 Bolsa Avenue

Huntington Beach, California 92647

George D. Rohades

Lockeed

Department 6263

Building 104

Jumyvale, Callfornia 94086

Universidad Nacional Autonoma

de Mexico

Instituto de Ingenieria

APDO, FQ 472

MEXICO, D.F.

Attn: Luis Palacios 


\begin{tabular}{|c|c|c|c|}
\hline \multirow[t]{2}{*}{1800} & R. S. & Claassen & \\
\hline & Attn: & $\begin{array}{lll}1810 & \text { R. G. } \\
1830 & \text { M. } & \text { J. } \\
1840 & \text { N. }\end{array}$ & $\begin{array}{l}\text { Kepler } \\
\text { Davis } \\
\text { Magnani }\end{array}$ \\
\hline \multirow[t]{4}{*}{1820} & R. E. & Whan & \\
\hline & Attn: & 1821 N. E. & Brown \\
\hline & & 1822 K. H. & Eckelmeyer \\
\hline & & 1823 J. A. & Borders \\
\hline 1824 & J. N. & Sweet & \\
\hline 1824 & E. P. & Roth & \\
\hline 1824 & R. B. & Pettit (4) & \\
\hline 1824 & A. $R_{\text {. }}$ & Mahoney & \\
\hline 8214 & M. A. & Pound & \\
\hline 0310 & D. M. & Eahustor & \\
\hline 8450 & c. s. & Selvage (2) & \\
\hline 8452 & w. s. & Rorke & \\
\hline 8452 & E. T. & Cull & \\
\hline 8452 & A. F. & Baker & \\
\hline 9721 & J. F. & Banas & \\
\hline 9721 & G. W. & Treadwell & \\
\hline$y / 21$ & L. M. & Larsen & \\
\hline 9722 & J. V. & otts & \\
\hline 9722 & J. T. & Holmes & \\
\hline 9724 & E. C. & Boes & \\
\hline 9724 & D. L. & King & \\
\hline 3141 & L. J. & Erickson (5) & \\
\hline 3151 & W. L. & Garner (3) & \\
\hline $31 \zeta 4$ & c. Lail & $\operatorname{lin}(25)$ & \\
\hline & for $D C$ & DE/TIC & \\
\hline
\end{tabular}




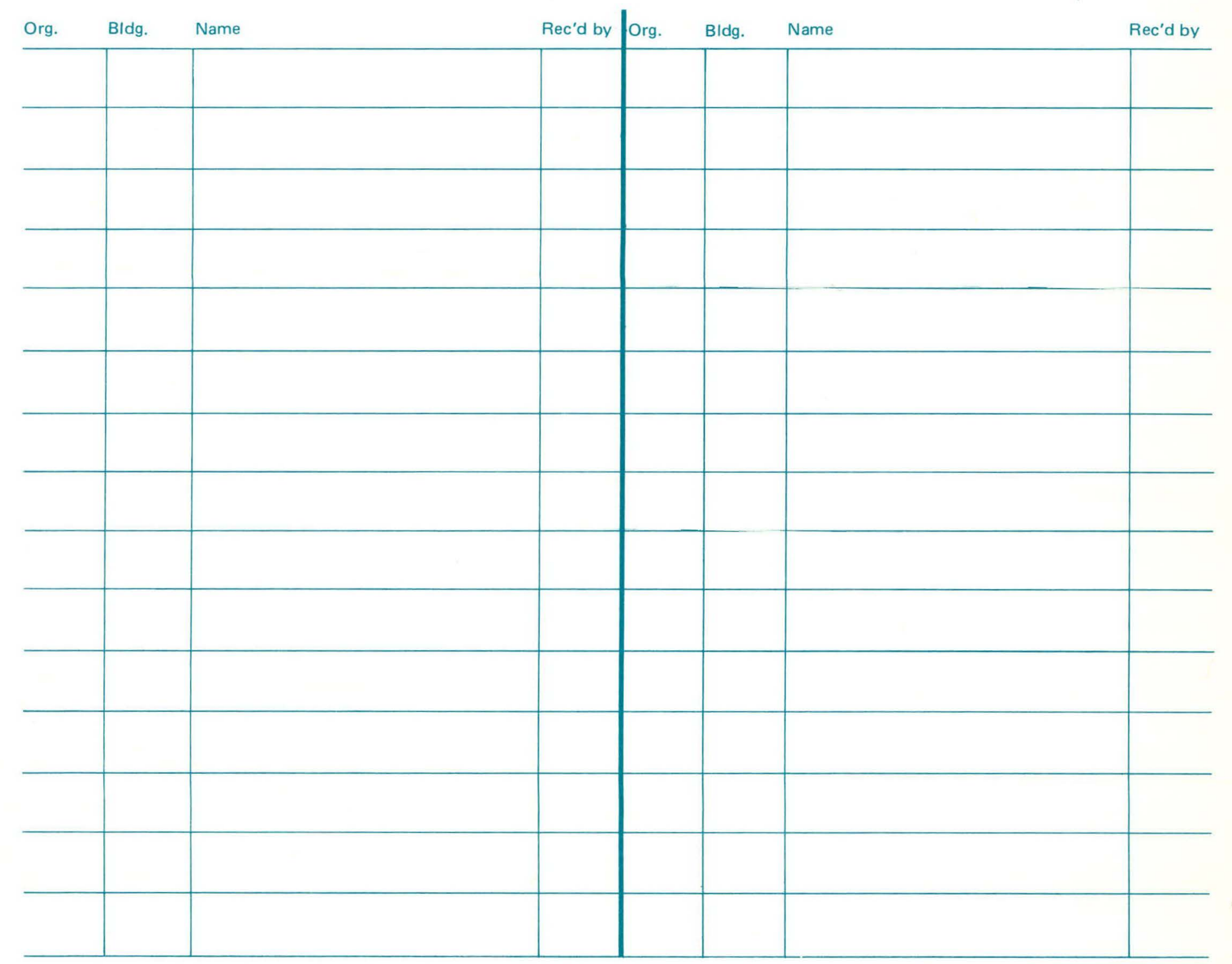

